\title{
List of Notations
}

\section{Operations and Symbols}

$:=$
$\langle\cdot, \cdot\rangle$
$\times$
$x \rightarrow \bar{x}$
$x \stackrel{A}{\rightarrow} \bar{x}$
$x \stackrel{f}{\rightarrow} \bar{x}$
$x_{n} \rightarrow \bar{x},\left(x_{n}\right) \rightarrow \bar{x}$
$x_{n} \stackrel{A}{\rightarrow} \bar{x}$
$\lim$
$\liminf$
$\lim \sup$
$\|\cdot\|$
$|\cdot|$

\section{Sets and Spaces}

$\emptyset$
$\mathbb{N}$
$\mathbb{N}^{\star}$
$\mathbb{Z}$
$\mathbb{Q}$
$\mathbb{R}$
$\mathbb{R}^{p}, p \in \mathbb{N}^{\star}$
$\mathbb{R}_{+}^{p}$
$B(x, r)$
$D(x, r)$
$S(x, r)$
$\operatorname{int} A$
$\operatorname{cl} A$
$\operatorname{bd} A$

equal by definition

scalar product

cartesian product

$x$ converges to $\bar{x}$

$x \rightarrow \bar{x}$ with $x \in A$

$x \rightarrow \bar{x}$ with $f(x) \rightarrow f(\bar{x})$

sequence $\left(x_{n}\right)$ has the limit $\bar{x}$

$x \rightarrow \bar{x}$ with $\left(x_{n}\right) \subset A$

limit (for sequences or functions)

lower limit

upper limit

Euclidean norm of $\mathbb{R}^{p}, p>1$

modulus (on $\mathbb{R}$ )

end of proof/solution 
$\operatorname{conv} A$

cone $A$

$A^{-}$

$T_{B}(A, \bar{x})$

$T_{C}(A, \bar{x})$

$N(A, \bar{x})$

$N_{B}(A, \bar{x})$

$N_{C}(A, \bar{x})$

$N_{F}(A, \bar{x})$

$N_{M}(A, \bar{x})$

$\partial f(\bar{x})$

$\partial_{C} f(\bar{x})$

$\partial_{F} f(\bar{x})$

$\partial_{M} f(\bar{x})$

$\partial^{\infty} f(\bar{x})$

$\operatorname{pr}_{A} \bar{x}$

$\operatorname{rank} A$

$A^{t}, x^{t}$

$\operatorname{dim} X$

\section{Functions}

$f: A \rightarrow B$
$f^{-1}$
$f \circ g$
$\operatorname{gr} f$
epi $f$
$N_{v} f$
$\operatorname{dom} f$
$\operatorname{Ker} T$
$\operatorname{Im} f$
$\nabla f(\bar{x})$
$\nabla^{2} f(\bar{x})$
$f^{\star}$
$d_{A}, d(\cdot, A)$
$\Delta_{A}$
$s_{e}$

convex hull of the set $A$

conic hull of the set $A$

polar of the set $A$

Bouligand tangent cone to $A$ at $\bar{x}$

Clarke tangent cone to $A$ at $\bar{x}$

normal cone to the convex set $A$ at $\bar{x}$

Bouligand normal cone to $A$ at $\bar{x}$

Clarke normal cone to $A$ at $\bar{x}$

Fréchet normal cone to $A$ at $\bar{x}$

Mordukhovich normal cone to $A$ at $\bar{x}$

convex subdifferential of $f$ at $\bar{x}$

Clarke subdifferential of $f$ at $\bar{x}$

Fréchet subdifferential of $f$ at $\bar{x}$

Mordukhovich subdifferential of $f$ at $\bar{x}$ singular subdifferential of $f$ at $\bar{x}$

projection set of $\bar{x}$ on $A$

rank of the matrix $A$

transpose of the matrix $A$ /vector $x$

dimension of the linear (sub)space $X$

function from $A$ to $B$

inverse of $f$

composition of functions

graph of $f$

epigraph of $f$

$v$-level set of $f$

domain of $f$

kernel of the linear operator $T$

image of the mapping $f$

gradient of $f$ at $\bar{x}$

second order differential of $f$ at $\bar{x}$

conjugate of $f$

distance function to the set $A$

oriented distance function to the set $A$

Gerstewitz (Tammer) scalarization functional 